because other organisms also have panoplies of sugar porters. For example, such proteins are used by plant cells to accumulate sugars into vacuoles (cytoplasmic vesicles) ${ }^{10}$, and they regulate sugar fermentation in yeasts during the production of alcohol from grape juice ${ }^{11}$. Moreover, some proteins that transport molecules other than sugars also possess the sugarporter signature sequences. This is the case for plant PHT1 phosphate transporters ${ }^{12}$, which not only take up the vital nutrient phosphate from the soil but also regulate its distribution throughout the plant. Sun and colleagues' paper is therefore likely to have ramifications in fields ranging from medicine to agriculture and the production of food and drink.

Peter J. F. Henderson and Stephen

A. Baldwin are in the Astbury Centre

for Structural Molecular Biology,
School of Biomedical Sciences,

University of Leeds, Leeds LS2 9JT, UK.

e-mail:p.j.f.henderson@leeds.ac.uk

1. Nicholson, D. Metabolic Pathways 22nd edn (2003); www.iubmb-nicholson.org/index.html

2. Sun, L. et al. Nature 490, 361-366 (2012).

3. Maiden, M. C. et al. Nature 325, 641-643 (1987).

4. Pao, S. S., Paulsen, I. T. \& Saier, M. H. Microbiol. Mol. Biol. Rev. 62, 1-34 (1998).

5. Jardetzky, O. Nature 211,969-970 (1966)

6. Boudker, O. \& Verdon, G. Trends Pharmacol. Sci. $\mathbf{3 1}$ 418-426 (2010)

7. Oka, Y. et al. Nature 345, 550-553 (1990).

8. Weinglass, A. B. \& Kaback, H. R. Proc. Natl Acad. Sci. USA 97, 8938-8943 (2000)

9. Thorens, B. \& Mueckler, M. Am. J. Physiol. Endocrinol. Metab. 298, E141-E145 (2010)

10.Slewinski, T. L. Mol. Plant 4, 641-662 (2011).

11. Kruckeberg, A. L. \& Dickinson, J. R. in The Metabolism and Molecular Physiology of Saccharomyces cerevisiae (eds Dickinson, J. R. \& Schweizer, M.) 42-103 (2004).

12. Nussaume, L. et al. Front. Plant Sci. 2, 83 (2011).

HIV

\title{
Design by trial
}

\section{A genetic analysis of viruses infecting participants in an HIV vaccine trial indicates that the vaccine is more protective against viruses that have variations at specific sites in the viral envelope. SEE LETTER P.417}

\section{DAVID V. GLIDDEN}

$\mathrm{T}$ he past five years have brought stunning progress in the development of biomedical approaches to preventing HIV infection. But despite recent hope for an AIDS-free generation, we still lack a crucial tool needed to reach this goal - an HIV vaccine. However, on page 417 of this issue, Rolland et al. ${ }^{1}$ present an analysis of a clinical trial that encourages us to believe that this goal is achievable. Furthermore, their work identifies a site on the virus's outer coat as a key target for future vaccine development.

Until a few years ago, the handful of candidate HIV vaccines that had been evaluated in randomized clinical trials had all failed to prevent HIV infections any better than a placebo. But in 2009, researchers conducting a trial in 16,402 volunteers in Thailand reported ${ }^{2}$ a $31 \%$ reduction in HIV infections in recipients of a vaccine known as RV144. The study's results were met with a range of responses, from cautious optimism to outright scepticism ${ }^{3}$. Although RV144 had some efficiency, this was modest. For example, the credible range for protection from infection elicited by the vaccine ran between $1 \%$ and $51 \%$ - a very wide range, and negligible at its low end. In addition, sensitivity analyses of the trial results failed to cross the threshold of statistical significance, and some experts openly speculated that the outcome was a statistical fluke.
A recent review ${ }^{4}$ that formally combined the trial's findings and expert opinion estimated at least a $22 \%$ chance that the reported outcome was a false positive.

In fact, doubts about RV144 had been expressed even before the study started ${ }^{5}$. The concerns stemmed from the fact that RV144 is a 'prime and boost' vaccine composed of two vaccines that had both shown only modest to no efficacy in previous studies. A trial of RV144 had been contemplated but scrubbed by the US-based HIV Vaccine Trials Network, and some informed observers had called for the Thai trial to be cancelled as well ${ }^{5}$. Despite this, it went ahead, and in the intervening three years, follow-up analyses of immune responses ${ }^{6}$ and now, from Rolland and colleagues, of HIV infections in the trial participants, are supporting an interpretation that RV144 was indeed successful at stimulating protective immunity in some of the vaccinated individuals.

Several candidate HIV vaccines, including RV144, contain proteins that belong to the outer coat or 'envelope' of HIV, with the intention of inducing protective immune memory against this region of the virus. However, the amino-acid sequence of the envelope varies among different circulating HIV viruses, so that any single vaccine based on this structure may not stimulate immune recognition across all HIV variants.

Rolland et al. examined the sequences of the envelope protein of HIV viruses isolated from people who became infected during the trial, to deduce the effectiveness of the vaccine against different viruses. In principle, if the vaccine has no effect on a particular virus type, then that virus should be found in roughly equal proportions in infected people who had received the vaccine and in those given the placebo. The researchers focused their typing on the V1/V2 loop structure of the viral envelope protein, a region previously implicated as being important for immune protection by a study showing that immune responses involving antibodies against $\mathrm{V} 1$ and $\mathrm{V} 2$ are associated with reduced risk of HIV infection ${ }^{6}$. Preliminary work identified 15 sites along the V1/V2 loop as specific targets of these putative protective antibodies, and Rolland and colleagues made these sites the subject of their sequence analysis.

The authors found two 'genetic signatures' in the V2 region - an amino-acid match between virus and vaccine at position K169 (lysine) and a mismatch at position I181 (isoleucine in the vaccine sequence) - that were proportionally less common in viruses that had infected vaccinated individuals compared with recipients of the placebo. This suggests that the vaccine blocked or 'sieved out' viruses with these sequence features, although this effect was variable: the vaccine had $48 \%$ efficacy at preventing infection with viruses matching at K169 and 78\% efficacy against viruses with a mismatch at I181. This differential effect of the RV144 vaccine in regard to HIV type is compelling, if indirect, evidence that the vaccine elicited immune responses that protected some people against some variants of HIV. Furthermore, because both sites are on the $\mathrm{V} 2$ region, the findings implicate this sequence as a key target for future vaccine development.

In other respects, however, the results defy a simple narrative. First, in the case of the K169 position, the vaccine protects best against viruses with the same sequence as that in the vaccine. Given that a vaccine is expected to 'train' the immune system, this result is unsurprising. However, the opposite is the case for position I181, and the reason for this is unclear. It is not that the vaccine promoted infection with viruses that matched it at I181; the rate of infection with such variants was still lower in vaccine recipients than in people receiving the placebo. It is possible, however, that I181 is merely a proxy for another, unidentified, location on $\mathrm{V} 2$ that does indeed match between the vaccine and these viruses.

Second, the results do not square neatly with the recent finding that the presence of antibodies against V1 and V2 was correlated with a reduced risk of HIV infection in those receiving the vaccine ${ }^{6}$. This association seems to be similar for vaccine-matching and non-matching viruses, but if it is the antibodies that provide the 'sieve' effect reported by Rolland and colleagues, one would expect 
them to be less protective against strains for which the vaccine was less effective. So although the sieve and correlates-of-protection analyses are both compelling, these two pieces of the story do not fully explain each other.

Although such questions remain open, Rolland and colleagues' study is another demonstration of the indispensable role of randomized trials in the incremental process of HIV vaccine development. The authors' research exemplifies how data from such trials can provide information on strain-specific effects and HIV-protective immune responses, and their analysis has identified an area on the HIV envelope that will be a focus of future vaccine studies. This comes at a time of steady progress in HIV vaccine research ${ }^{7}$ and buoys up the hope that an HIV vaccine is possible and perhaps sooner than later.

David V. Glidden is in the Department of Epidemiology and Biostatistics, University of California, San Francisco, San Francisco, California 94107, USA.

e-mail:dave@biostat.ucsf.edu

1. Rolland, M. et al. Nature 490, 417-420 (2012).

2. Rerks-Ngarm, S. et al. N. Engl. J. Med. 361, 2209-2220 (2009)

3. Leavy, O. Nature Rev. Immunol. 9, 755 (2009).

4. Gilbert, P. B. et al. J. Infect. Dis. 203, 969-975 (2011)

5. Burton, D. R. et al. Science 303, 316 (2004).

6. Haynes, B. F. et al. N. Engl. J. Med. 366, 1275-1286 (2012).

7. Wu, X. et al. Science 329, 856-861 (2010).

\section{Choose your protein partners}

\begin{abstract}
A large-scale study sheds light on the extraordinary molecular-recognition skills of the chaperone HSP90, which allow this protein to interact selectively with hundreds of other proteins of diverse function.
\end{abstract}

\section{RAHUL S. SAMANT \& PAUL WORKMAN}

$\mathrm{T}$ he protein HSP90 plays an essential part in a plethora of cellular processes, in evolution and in disease. It is a molecuactivity and stability of other proteins, which are known as its 'clients'. Many of HSP90's clients are oncogenic protein kinases - when made overactive, they can lead to cancer ${ }^{1}$. That are currently in clinical trials for antitumour therapy ${ }^{2}$. Despite all this interest, the fundamental question of how HSP90 chooses its clients remains unanswered. Writing in Cell, Taipale et al. ${ }^{3}$ report that another protein (a cochaperone) facilitates client-family recognition by HSP90, whereas the thermal stability of the client determines the strength of its interaction with the chaperone itself.

In the absence of functional HSP90, its clients form aggregates or are degraded. So previous large-scale efforts to identify clients were based on either isolation of HSP90binding proteins $s^{4}$ or demonstration of client depletion in cells in which HSP90 function had been perturbed (for example, by pharmacological inhibition) $)^{5}$. These studies have so far revealed around 350 highly diverse clients for HSP90.

Taipale et al. carried out their own largescale survey of HSP90 clients among selected protein classes, including the protein kinases. They used a modification of the LUMIER assay ${ }^{6}$, a luminescence-based measure of association between a 'prey' protein (in this case, HSP90) and 'baits' (putative clients) in mammalian cells. Compared with other techniques used to detect binding, such as mass lar chaperone, a type of protein required for the is why 20 small-molecule HSP90 inhibitors spectrometry, the modified LUMIER assay is much more sensitive, detects shorter-lived interactions and gives a quantitative rather than a binary (binding/no binding) readout. Of the 314 kinases investigated, 193 (61\%) interacted to some extent with HSP90. Strikingly, the strength of these interactions varied across a continuous 100 -fold range. This finding supports the growing view that the binary categorization of kinases into clients or non-clients should be replaced by a continuum of HSP90 dependence.

In agreement with an earlier analysis of binary data ${ }^{7}$, Taipale and colleagues confirmed and extended previous findings that even very closely related kinases (such as ARAF and BRAF) exhibit extreme differences in the strength of their interactions with HSP90 (refs 1,8). Seeking a structural explanation for this, Taipale et al. constructed and analysed variants of ARAF and BRAF containing singlepoint mutations, and also analysed chimaeric proteins made of different pieces taken from the two kinases. Notably, no single amino-acid change within the catalytic kinase domain (the part of the protein that defines a protein kinase) altered the protein's affinity for HSP90. Rather, the authors found that determinants for HSP90 association were widely distributed across the kinase domain.

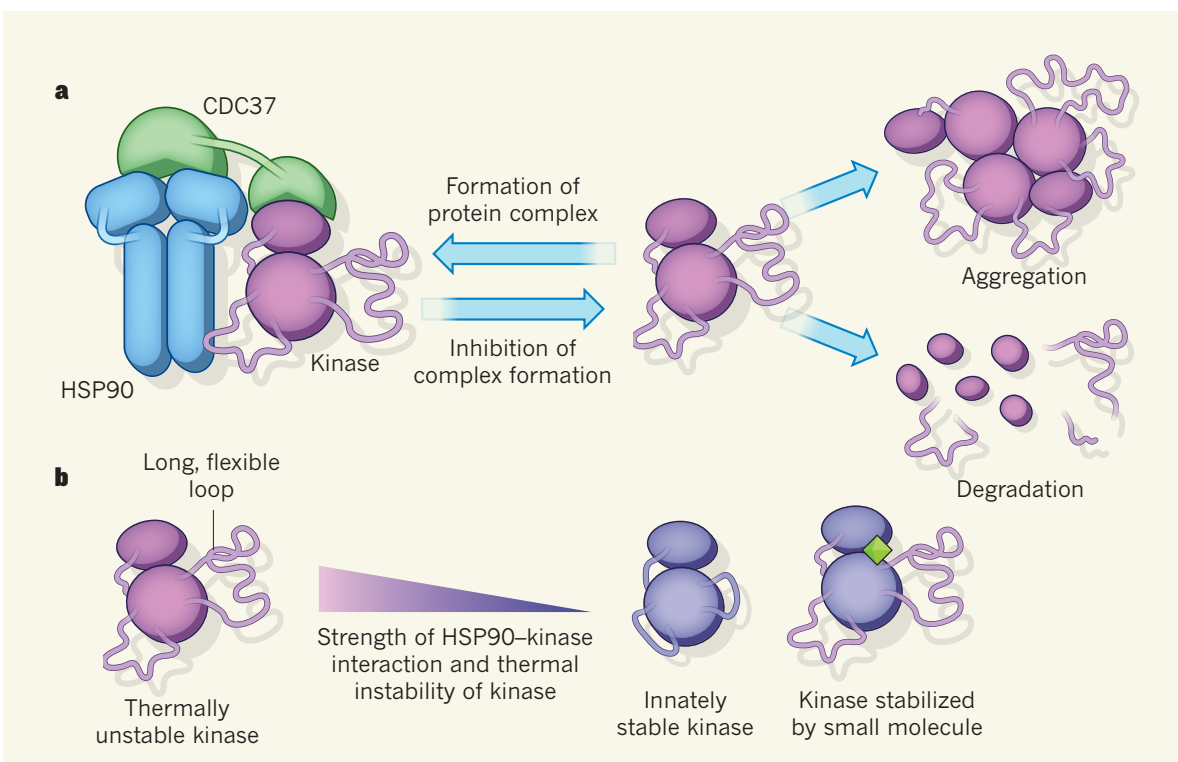

Figure 1 | A helping hand for unstable proteins. a, Many proteins of the protein-kinase family form aggregates or are degraded unless they form a complex with the chaperone protein HSP90. Binding of the kinase to HSP90 can be reversed by the use of HSP90 inhibitors, for example. Taipale et al. ${ }^{3}$ report that another protein, co-chaperone CDC37, facilitates the recognition of kinase-family features by HSP90. b, The authors also show that the strength of the HSP90-kinase interaction is correlated with the thermal instability of the kinase. Some of this instability might be due to the presence of long, highly flexible loops in the kinase domain, because protein-kinase variants possessing certain shorter flexible segments display increased thermal stability and decreased interaction with HSP90. Small molecules stabilized the kinases and led to weaker association with HSP90. 